\title{
Computed tomography-guided simultaneous coil localization of multiple pulmonary nodules before video-assisted thoracoscopic surgery
}

\author{
Min Ai, Jian Xu \\ Department of Interventional Therapy, Jinling Hospital, Clinical School of Medical College, Nanjing University, Nanjing, Jiangsu Province, \\ China
}

Videosurgery Miniinv 2022; 17 (1): 245-251

DOI: https://doi.org/10.5114/wiitm.2021.105683

\begin{abstract}
Introduction: Currently, different methods and materials are used to localize pulmonary nodules (PNs) but most are used only to locate a single pulmonary nodule (PN).

Aim: To evaluate the feasibility and safety of simultaneously localizing multiple PNs with a coil under computed tomography (CT) guidance before video-assisted thoracoscopic surgery (VATS).

Material and methods: A total of 166 patients underwent VATS preoperative-assisted localization of pulmonary nodules in our hospital in the period from January 2, 2020 to July 7, 2020, namely 40 patients in the multiple-PN-simultaneous-localization group $(A)$ and 126 patients in the single- $P N$-localization group $(B)$. We compared the epidemiology, localization procedure, and complications between the two groups.

Results: The technical success rates in group $A$ and Group B were $96.5 \%$ and $97.6 \%$, respectively, with no statistical difference ( $p=0.623)$. In group $A$, the success rate of the first nodule localization was $100 \%$, and the subsequent nodule localization success rate was 93.3\%; 3 patients had one nodule localization failure owing to pneumothorax after the first nodule localization. The number of pleural punctures was higher in group $A$ than in group $B$ ( $p<0.001)$, and the localization procedure time was longer than in group $B(p<0.001)$. Regarding complications, the pneumothorax rate in group $A$ was higher than that in group $B(p<0.001)$, and the bleeding rate was higher than that in group $B(p<0.001)$. However, pneumothorax and bleeding in group $A$ did not require special treatment. Conclusions: The incidence of pneumothorax and pulmonary hemorrhage with simultaneous coil localization of multiple PNs was higher than that with localization of a single PN, but this method was safe and feasible.
\end{abstract}

Key words: coil localization, pulmonary nodules, multiple, computed tomography.

\section{Introduction}

With the popularization of computed tomography $(\mathrm{CT})$, the detection rate of pulmonary nodules (PNs) $(<10 \mathrm{~mm})$ has improved greatly [1]. There is a difference between the $\mathrm{CT}$ appearance and the pathological diagnosis of PNs, and the positive rate of PN puncture biopsy is not high, especially for ground-glass-opacity (GGO) lesions [2]. Diagnostic wedge resection is currently the accepted standard method for managing PNs, while video-assisted thoracic surgery (VATS) is widely used for the diagnostic resection of PNs owing to the advantages of less trauma, less pulmonary function injury, and fewer complications [3].

When the lesion is deep in the pleural surface or has a ground-glass density, it is difficult to detect the

\section{Address for correspondence}

Jian Xu, Department of Interventional Therapy, Jinling Hospital, Clinical School of Medical College, Nanjing University, Nanjing 210002,

Jiangsu Province, China, e-mail: 13851656307@163.com 
lesion during VATS, which affects the success rate of VATS [4]. Preoperative VATS-assisted localization of PNs can significantly improve the success rate of PN resection [5]. Currently, different methods and materials are used to localize PNs but most are used only to locate a single PN [6-8]. Clinically, it is common for patients to have multiple PNs, such as multiple orthotopic carcinoma or metastatic carcinoma. Simultaneous excision of multiple $\mathrm{PNs}$ can reduce the risk of disease progression during the interval between staging operations, shorten the treatment time, and reduce the patient's economic burden [9]. As with the localization of a single PN, simultaneous localization of multiple PNs can improve the PN resection rate, but simultaneous assisted localization of multiple PNs before VATS is rarely reported.

In this study, the feasibility and safety of simultaneously localizing multiple PNs with a coil under CT guidance were evaluated by comparing a multiple-PN-simultaneous- with a single-PN-localization group.

\section{Aim}

To evaluate the feasibility and safety of simultaneously localizing multiple PNs with a coil under CT guidance before VATS.

\section{Material and methods}

\section{Patients}

We evaluated 166 patients who underwent VATS preoperative-assisted localization of PNs in the period from January 2, 2020 to July 7, 2020 in our hospital. The multiple-PN-simultaneous-localization group (group A) constituted 40 patients with a total of 85 nodules. The single-PN-localization group (group B) constituted 126 patients with a total of 126 nodules. All patients who underwent VATS pre-operatively for assisted localization provided signed informed consent. Because this was a retrospective study, ethical approval from our institution was not required. We collected patients' demographic data, lesion characteristics, localization procedure, and length of hospital stay for both groups, and then statistically analyzed the data.

All patients in this study had nodules located in the ipsilateral lung. The lesion location in the lung was divided into the upper or middle lobe, and the lower lobe. The nodule type was divided into pure GGO (pGGO), solid nodules, and mixed GGO
(mGGO). pGGO indicates a slight increase in density with fuzzy local lung tissue, but this difference does not affect the visibility of the bronchi and vascular bundles. Solid nodules are lesions with increased density and complete occlusion of bronchi and vascular bundles. mGGO indicates a lesion containing both solid and ground-glass opacity features.

\section{Localization procedures}

Before the nodule localization operation, we analyzed the patient's chest CT images after admission to understand where the nodules were located in the lung(s), and then determined the optimal puncture position for the patient in advance. After positioning the patient on the CT scan bed in the pre-determined position, and using the scanned images, we determined the priority of locating several nodules and the optimal puncture route for each nodule.

Local anesthesia was achieved by injecting subcutaneous lidocaine into the pre-determined injection point. We used the following two methods to insert the 18-G 150-mm puncture needle (PTC) (Hakko Co., Ltd.) next to the lesion according to the pre-determined puncture route: A. For lesions close to the pleural surface, the needle tip was inserted directly and fully into the adjacent lesion in one motion. B. For lesions far from the pleural surface, to prevent the tip from deviating from the lesion after a single insertion, the PTC needle was inserted to half the distance of the optimal puncture route after the needle tip punctured the pleura into the lung (this ensures that subsequent needle insertions will not puncture the pleura again and increase the incidence of pneumothorax). Then, CT was performed to determine the relationship between the needle tip and the lesion location; the insertion angle of the remaining distance of the insertion distance was adjusted accordingly; and we advanced the needle tip next to the lesion.

After repeating the $\mathrm{CT}$ scans to confirm that the PTC tip was inserted near the lesion, we retracted the inner needle from the PTC, then inserted a 0.035 inch diameter, 30-mm long coil (Cook Medical) into the sheath of the PTC, advanced the coil through the sheath to the tip with the inner needle in the PTC, and moved the inner needle back and forth several times to prevent the end of the coil from remaining in the sheath of the PTC. Finally, CT was performed to confirm that the coil was beside the lesion, then 
the PTC was removed (Photos $1 \mathrm{~A}, \mathrm{~B}$ ). The posterior PNs (Photos 1 C, D) were located in the same manner until all nodules had been located and then the localizing procedure was completed.

\section{Statistical analysis}

Continuous variables are expressed as mean \pm standard deviation (SD). The $t$-test was used to analyze differences in the continuous variables between the two groups, namely age, maximum lesion diameter, distance from the lesions to the pleural surface, number of pleural punctures, positioning operation time, and length of hospital stay. The $\chi^{2}$ test was used to analyze the differences in data and rates between the two groups, namely sex, nodule type, lobar location of the nodule, success rate of the localization technique, incidence of pneumothorax, and bleeding.

\section{Results}

There were 40 patients in group $\mathrm{A}$, and 85 nodules were localized, namely two nodules in $36 \mathrm{pa}$ -
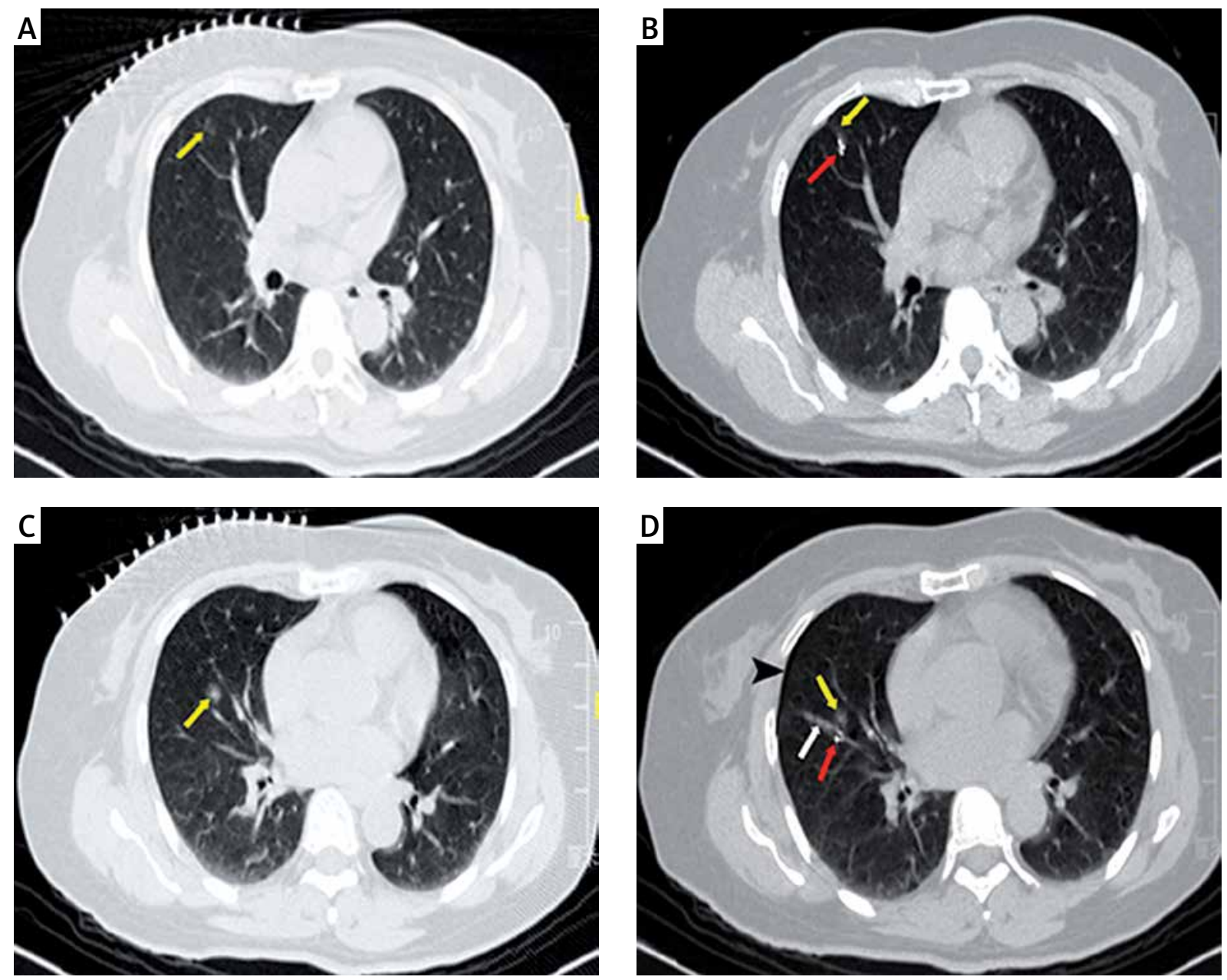

Photo 1. A 51-year-old woman presented with two lesions in her right lung. The patient underwent simultaneous coil localization for both lesions under CT guidance. A - The CT image shows a lesion in the upper lobe of the right lung (yellow arrow), with a diameter of $8.2 \mathrm{~mm}$ and a distance of $6.6 \mathrm{~mm}$ from the pleural surface. B - CT image showing a coil (red arrow) adjacent to the lesion in the upper lobe of the right lung (yellow arrow). C - CT image showing a lesion in the middle lobe of the right lung (yellow arrow), with a diameter of $5.0 \mathrm{~mm}$ and a distance of $34.5 \mathrm{~mm}$ from the pleural surface. D - CT image shows a coil (red arrow) located next to the lesion in the middle lobe of the right lung (yellow arrow), with a small amount of pneumothorax (black arrowhead) and pulmonary hemorrhage (white arrow) 
tients, three nodules in 3 patients, and four nodules in 1 patient. In group A, the first nodule was localized successfully (40/40), and subsequent nodules were localized in $93.3 \%$ of the patients (42/45) (Table I). Localizing the posterior nodule in 3 patients was affected by pneumothorax caused by localizing the first nodule, and localizing these 3 nodules eventually failed, as a result. All nodules in group A were successfully removed, and all coils were removed.

Table II shows the comparison of age, sex, lesion type, maximum lesion diameter, distance of the lesion from the pleura, location of the lesion in the lung lobe, and length of hospital stay between groups A and B. We found no statistically significant difference in baseline data between the two groups $(p>0.05)$. Most of the patients in both groups were women; most nodules were pGGO; nodules were located mainly in the upper or middle lobes; and the mean maximum lesion diameter in both groups was $<10 \mathrm{~mm}$.

Table III shows the comparison of the positioning operations between groups $A$ and $B$. The number of pleural punctures was higher in group $A$ than in group B (3.1 vs. 1.3 , respectively; $p<0.001)$, and the operation time was longer in group $A$ vs group $B$ (17.5 min vs. $8.3 \mathrm{~min}$, respectively). The incidence of pneumothorax was higher in group $A$ vs group $B$ (35\% vs. $12.7 \%$, respectively; $p<0.001$ ), and the bleeding incidence was higher (60\% vs. $31.7 \%$, respectively; $p<0.001$ ), but no significant difference in the technical success rate was found between the two groups (96.5\% vs. $97.6 \%$, respectively; $p=0.355)$. Pneumothorax induced by localization in group A was asymptomatic and did not require chest tube drainage. All the bleeding caused by positioning in group A was small-volume pulmonary

Table I. Characteristics of group A

\begin{tabular}{|lccc|}
\hline Variable & 2 nodules & 3 nodules & 4 nodules \\
\hline Patients $(n)$ & 36 & 3 & 1 \\
\hline Technical success $(+/-):$ & & $3 / 0$ & $1 / 0$ \\
\hline First nodules & $36 / 0$ & $6 / 0$ & $2 / 1$ \\
\hline Subsequent nodules & $34 / 2$ & & \\
\hline
\end{tabular}

Table II. Characteristics of the patients and lesions

\begin{tabular}{|lccc|}
\hline Characteristics & Group A & Group B & $P$-value \\
\hline Number of patients & 40 & 126 & 126 \\
\hline Number of lesions & 85 & & 0.089 \\
\hline Age [years]: & $55.0 \pm 9.8$ & $51.1 \pm 13.3$ & 0.106 \\
\hline Mean & $35-78$ & $20-83$ & 0.348 \\
\hline Range & $12 / 28$ & $56 / 70$ & 0.9 \\
\hline Sex (men/women) & $67 / 13 / 5$ & $88 / 28 / 10$ & 0.6 \\
\hline Type of lesion (pGGO/mGGO/solid) & & $9.8 \pm 3.2$ & 0.218 \\
\hline Maximum lesion diameter [mm]: & $9.7 \pm 3.0$ & $1.1-21.4$ & 0.823 \\
\hline Mean & $1.4-20.6$ & $13.0 \pm 10.9$ & $86 / 40$ \\
\hline Range & $12.0 \pm 8.8$ & $8.1 \pm 3.0$ & \\
\hline Distance from the pleura [mm] & $51 / 34$ & $8.0 \pm 2.7$ & \\
\hline Location (upper or middle lobe/lower lobe) & & & \\
\hline Hospital stay [days] & & & \\
\hline
\end{tabular}

pGGO - pure ground-glass opacity, mGGO - mixed ground-glass opacity. 
Table III. Characteristics of localization procedures

\begin{tabular}{|lccc|}
\hline Characteristics & Group A & Group B & $P$-value \\
\hline Number of pleural punctures & $3.1 \pm 1.1$ & $1.3 \pm 0.6$ & $<0.001$ \\
\hline Procedure time [min] & $17.5 \pm 6.0$ & $8.3 \pm 2.6$ & $<0.001$ \\
\hline Technical success rate: & $96.5 \%$ & & 0.623 \\
\hline Lesions & $92.5 \%$ & $97.6 \%$ & 0.131 \\
\hline Patients & $14 / 26$ & $97.6 \%$ & $<0.001$ \\
\hline Pneumothorax (+/-) & $24 / 16$ & $16 / 110$ & $<0.001$ \\
\hline Pulmonary hemorrhage $(+/-)$ & & $40 / 86$ & \\
\hline
\end{tabular}

hemorrhage, and some patients presented with small-volume $(10-20 \mathrm{ml})$ hemoptysis and recovered after a short rest without special treatment.

\section{Discussion}

The technical success rate of PN localization in group A in this study was $95.3 \%$ and $92.5 \%$ in lesionand patient-based analyses, respectively, and there was no difference compared with group B. Additionally, the success rate of PN localization in group $A$ in this study was consistent with that of previous studies of multiple PNs' localization [10-14]. These findings suggest that simultaneous coil localization of multiple PNs by CT guidance is effective. In this study, the average maximum lesion diameter in both groups was < $10 \mathrm{~mm}$, and most lesions were pGGO, suggesting that the role of the coil in localizing PNs helped surgeons to quickly and accurately identify small GG nodules during VATS and improve the wedge resection rate.

Pneumothorax and pulmonary hemorrhage are common complications of PN localization [4, 15]. In this study, both the pneumothorax rate $(30.5 \%)$ and bleeding rate $(60 \%)$ in group $A$ were higher than the rates in group $B$, which was related to the following circumstances. First, the number of pleural punctures in group A was significantly higher than that in group B, which increased contact between the thoracic cavity and air in vitro, or lung tissues, leading to pneumothorax. Second, the positioning operation time in group A was significantly longer than that of group B, but it was difficult for patients to maintain the puncture position for a long period; therefore, some patients moved a limb, which resulted in pneumothorax or bleeding. In this study, pneumothorax in group A was asymptomatic and did not require chest tube drainage. Additionally, all the bleeding was a small volume of hemorrhage in the lung, and occasionally, patients developed intraoperative hemoptysis, which was relieved after rest. These findings suggested that it was safe to use coils to locate multiple PNs during the same procedure under CT guidance.

The incidence of pneumothorax with simultaneous coil localization of multiple PNs under CT guidance was previously reported to be 12.9-15.8\% $[10,11]$, which is lower than the rate in our study (30.5\%). Through a comparative analysis, we found that the mean distance of the lesions from the pleural surface in Group A in this study was significantly greater than that in previous studies, and the incidence of pneumothorax increased with increased nodule depth [16]; therefore, the pneumothorax rate was relatively high in our study. In studies using hook wire localization for multiple PNs during the same procedure, the incidence of pneumothorax was 35.8-89.5\% [13, 15], which was higher than in our study. These previous studies reported a high incidence of pneumothorax requiring chest tube drainage, which prompted us to consider that coils may be a better choice in multiple-PN localization vs hook wire localization. The reasons for the difference in pneumothorax rates between the two techniques are as follows. First, the hook wire is easily moved into the thoracic cavity, resulting in pneumothorax. Second, the coil is coated with platinum and fibrin clots, which may reduce the risk of pneumothorax.

In this study, 3 patients in group A each had one nodule localization failure owing to pneumothorax after localizing the first nodule, suggesting that the priority of multiple PNs' localization should be discussed with the surgeon before the procedure. We recommend priority for nodules that must be local- 
ized (e.g., highly malignant nodules), and for nodules that the surgeon cannot detect during VATS.

Indocyanine green (ICG) has been used by some scholars to successfully locate PNs in 30 patients, and all lesions have been accurately resected [8]. ICG can be visually recognized by the near-infrared ray thoracoscope, and it does not need to be taken out after localization of PNs. At present, there is no control study on localization of PNs with a coil and ICG, so we cannot evaluate which material is more suitable for locating multiple PNs. However, simultaneous ICG localization of multiple PNs deserves our future study.

The simultaneous localization of multiple PNs in both lungs remains controversial. Some researchers have localized multiple PNs in both lungs simultaneously, which led to bilateral pneumothorax and the requirement for chest tube drainage; therefore, this localization approach is not recommended [13]. However, other researchers localized multiple PNs simultaneously in both lungs, and wedge-shaped resection of localized nodules in both lungs was successfully performed without adverse complications [10, 11]. In our study, there was no difference in the length of stay between group A and group B. However, if the nodules in group $A$ had been treated in stages, patients' hospitalization periods and economic burdens would have increased. Thus, simultaneous localization and resection of multiple PNs in both lungs before surgery may provide greater patient benefit. In this study, no patients underwent simultaneous localization of multiple PNs in both lungs, but our results provided a theoretical basis for performing subsequent localization of multiple PNs in both lungs.

This study had several limitations. First, this was a retrospective study, which involves selection bias. Second, in all patients in this study, multiple PNs were identified in the ipsilateral lung, but we did not perform simultaneous localization of multiple PNs in both lungs. Third, risk factor analysis of pneumothorax after simultaneous localization of multiple PNs was lacking in this study. Despite the above shortcomings, to our knowledge, the present study is the first to compare localizing multiple PNs with a single PN under CT guidance.

\section{Conclusions}

Simultaneous coil localization of multiple PNs increased the incidence of pneumothorax and pulmo- nary hemorrhage, but this approach was safe and feasible.

\section{Conflict of interest}

The authors declare no conflict of interest.

\section{References}

1. Horeweg N, van Rosmalen J, Heuvelmans MA, et al. Lung cancer probability in patients with CT-detected pulmonary nodules: a prespecified analysis of data from the NELSON trial of lowdose CT screening. Lancet Oncol 2014; 15: 1332-41.

2. Yeow KM, Tsay PK, Cheung YC, et al. Factors affecting diagnostic accuracy of CT-guided coaxial cutting needle lung biopsy: retrospective analysis of 631 procedures. J Vasc Interv Radiol 2003; 14: 581-8.

3. Naidich DP, Bankier AA, MacMahon H, et al. Recommendations for the management of subsolid pulmonary nodules detected at CT: a statement from the Fleischner Society. Radiology 2013; 266: 304-17.

4. Ichinose J, Kohno T, Fujimori S, et al. Efficacy and complications of computed tomography-guided hook wire localization. Ann Thorac Surg 2013; 96: 1203-8.

5. Finley RJ, Mayo JR, Grant K, et al. Preoperative computed tomography-guided microcoil localization of small peripheral pulmonary nodules: a prospective randomized controlled trial. J Thorac Cardiovasc Surg 2015; 149: 26-31.

6. Gu T, Yu JY, Guo T, et al. A novel CT-guided technique using medical adhesive for localization of small pulmonary ground-glass nodules and mixed ground-glass nodules ( $\leq 20 \mathrm{~mm}$ ) before video-assisted thoracoscopic surgery. Diagn Interv Radiol 2018; 24: 209-12.

7. Park $\mathrm{CH}$, Han K, Hur J, et al. Comparative effectiveness and safety of preoperative lung localization for pulmonary nodules: a systematic review and meta-analysis. Chest 2017; 151: 316-28.

8. Zhong L, Hu W, Li S, et al. Clinical study of video-assisted thoracoscopic surgery wedge resection in early-stage lung cancer by tumor mapping with indocyanine green. Videosurgery Miniinv 2019; 14: 545-50.

9. Yao F, Yang H, Zhao H. Single-stage bilateral pulmonary resections by video-assisted thoracic surgery for multiple small nodules. J Thorac Dis 2016; 8: 469-75.

10. Fu YF, Gao YG, Zhang M, et al. Computed tomography-guided simultaneous coil localization as a bridge to one-stage surgery for multiple lung nodules: a retrospective study. J Cardiothorac Surg 2019; 14: 43.

11. Teng F, Wu AL, Yang S, et al. Preoperative computed tomography-guided coil localization for multiple lung nodules. Ther Adv Respir Dis 2020; 14: 1753466620909762.

12. Tseng YH, Lee YF, Hsieh MS, et al. Preoperative computed tomography-guided dye injection to localize multiple lung nodules for video-assisted thoracoscopic surgery. J Thorac Dis 2016; 8: S666-71.

13. Iguchi T, Hiraki T, Gobara H, et al. Simultaneous multiple preoperative localizations of small pulmonary lesions using a short hook wire and suture system. Cardiovasc Interv Radiol 2015; 38: 971-6. 
14. Kadeer X, Wang L, Zhang L, et al. Modified hook-wire placement technique for localizing multiple pulmonary nodules. J Surg Oncol 2018; 118: 1188-93.

15. Zhong Y, Xu XQ, Pan XL, et al. Retrospective evaluation of safety, efficacy and risk factors for pneumothorax in simultaneous localizations of multiple pulmonary nodules using hook wire system. Cardiovasc Intervent Radiol 2017; 40: 1408-14.

16. Lim WH, Park CM, Yoon SH, et al. Time-dependent analysis of incidence, risk factors and clinical significance of pneumothorax after percutaneous lung biopsy. Eur Radiol 2018; 28: 1328-37.

Received: 3.01.2021, accepted: 15.02.2021. 